\title{
The Construction Principle of Double Cloth and Its Properties
}

\author{
Shilpi Akter* and Sutapa Chowdhury \\ Bangladesh University of Textiles, Bangladesh \\ *Corresponding author: Shilpi Akter, Bangladesh University of Textiles, Bangladesh, Email: shilpiakt@gmail.com \\ submission: 眥 January 18, 2018; Published: 望 February 08, 2018, $2^{\text {nd }}$ Edtion: 16 November, 2018
}

\begin{abstract}
The aim of this research paper is to produce woven cloth with good hand feel and with enhanced thermal resistance property. The construction of double cloth is useful where the development of thermal insulation value of a fabric with a fine appearance is essential. Double weaves are categorized by harness floats in paired or greater combinations on a face and altered paired or greater float combinations on the back. Double cloth is aesthetic in intension for that purpose the existence of two series of threads in every way develops the capacity for creating complicated properties depending upon both colour and structural variations. In double weave, the two cloths are connected where they change faces to form pattern generally in one colour against background. Air permeability, thermal conductivity and some other properties are tested and discussed in this research which provides and comfortability to the wearer of double cloth. Loose construction showed higher air permeability those were useful for the body. Compact fabrics with compact structure show less thermal conductivity values which resists the temperature to pass fast so that wearer will feel warm. Denser constructions showed less shrinkage with higher strength due to compactness in the structures.
\end{abstract}

Keywords: Double cloth; Air permeability; Thermal conductivity; Tear; Tensile; Shrinkage

\section{Introduction}

There is a great importance of this study in woven fabric production that can be utilized while making a two layered cloth namely double cloth in which two or more sets of warps and one or more sets of wefts are interconnected to make a two-layered cloth. The movement of threads in between the layers allows complex patterns and surface textures. In modern textile manufacturing, double cloth is occasionally restricted to fabrics where two warps and three wefts are made up as two distinct fabrics which joined by the third or binding weft. This difference is not always made in double-woven fabrics where two warps and two wefts interlace to shape geometric pattern. Double cloth is also called doubled faced fabric as it does not have any specific face side or back side. It includes the blankets, satin ribbons, and interlinings. Modern use of this double weave fabric is in coats, furnishing fabrics, and some brocades. In the double weave structure, the two weaves are joined by the weft of the subordinate weave that passes either above or below the key weave to generate pattern. In stitched double cloth, the two weave structures are joined where particular warp threads of one of them weave with the weft of the other. The modest signs of double cloth utilize both a point diagram and the weft cross section diagram to demonstration alternations in between top and bottom. In diagram, the warp yarns may be signified by each end to allow easy visualizations. Numerous recurrences of each end weaving pattern join to allow the double face of a fabric. A subtype of double weaves are velvets, which are designed with a travelling binder warp that is cut after weaving to form a pile on the surface of either fabric base.
The weaving of double cloth is an antique practice that are inherently doing through antecedent. The historical background of double weaving has been found dating back as from early 700AD. William Morris, the Craftsman and designer was well known for using double cloth in his intricate pieces of fabric [1]. That time, those were customarily made up with silk and wool with heavily patterned. These were fashioned in the late $19^{\text {th }}$ Century. Double cloth can be made up with wool, silk, cotton, viscose or polyester that depends upon the end use. Double cloth had separate warps of wool and silk yarn and were woven by Alexander Morton \& Co. of Darvel, Scotland, later who weave similar fabrics designs by C.F.A Voysey and others. Further thoughts in double weaves contain whether shuttle or shuttle less weaving methods are applied and whether immediate weft insertions in top and bottom sheds occur [2]. If shuttle weaving is employed, a nonstop loop of weft yarn is injected along crosswise the width of the fabric. Technical uses of this fabric type comprise vascular grafts and spacesuit joints. In the latter role, a double weave tubular fabric was established at Georgia Tech in the 1970s and 1980s by Dr. Howard Olson for elbow and knee joints as the first solution to avoid vascular devastation of astronauts' arms and legs through bending motions [3].

\section{Construction Principle of Double Cloth}

Actually, double cloths are the fabrics, which contains two layers of yarns those are woven one above the other and stitched together. Double cloths have at least two series of warp yarns, and two series of weft yarns, namely face and back. Double cloths fabrics are popularly known as two ply fabrics. The upper layer is formed 
by interlacing the face warp yarns with the face weft yarns, and the lower layer is formed by interlacing the back warp yarns with the back weft yarns. The two layers may be only loosely connected in which, each may be readily identified as a different entity, or they may be so intricately stitched or tied together that they appear to form a complex single structure [4]. Below Figure 1 shows a double cloth.

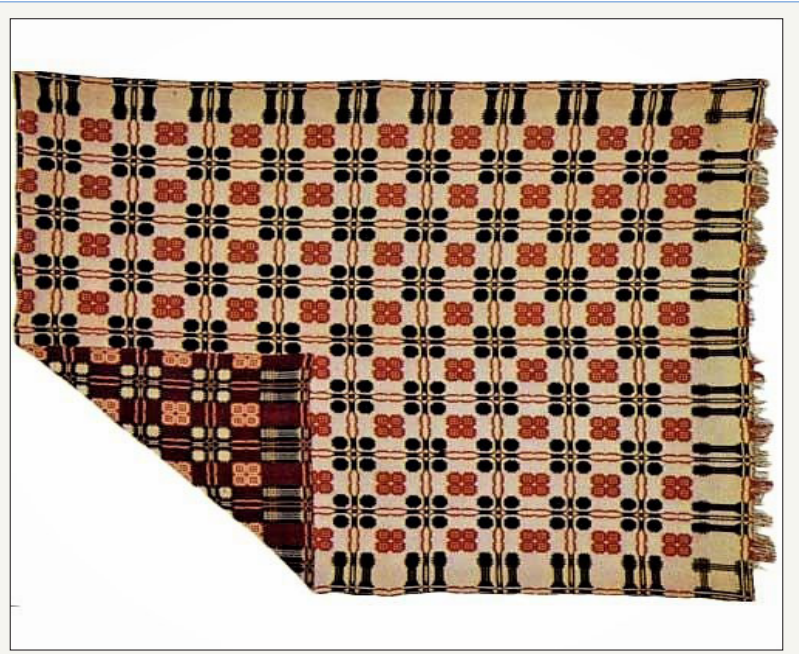

Figure 1: Double cloth.

Purpose of making double cloth:

i. To improve the thermal resistance value of the fabric

ii. To improve the air permeability of the fabric and

iii. To increase good appearance and hand feel of the fabric

Types of double cloth:

a) Self-stitched double cloth

b) Center stitched double cloth

c) Double cloth stitched by thread interchange

d) Double cloth stitched by cloth interchange

e) Alternate single ply and double ply construction

a) Self-stitched double cloth

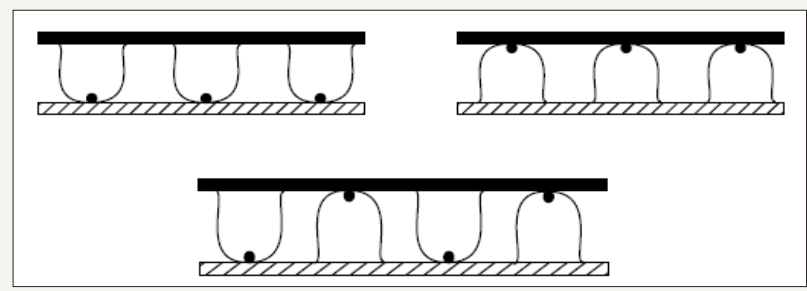

Figure 2: Self-stitched double cloth.

This double cloth is constructed on the principle of self-thread stitching. In this cloth, the face fabric is formed by the interlacement of the face warp and face weft threads and the back fabric is formed by the interlacement of the back warp and back weft threads. The two fabric layers are stitched together at intermediate points by either face/back warp or face/back weft or both. Distinctive structures with various possible techniques are drawn below. In a self-stitched double cloth fabric, one series of warp and weft interlace to form the face fabric and the other series of warp and weft interlace to form the back fabric. The face and back threads are supposed to be prearranged in an appropriate order, which depends on the fabric to be woven. Usually, different weaves are selected for the face and back fabrics. By the interlacement of the corresponding face threads, the face fabric is formed, the back fabric is [5]. Figure 2 showed a self-stitched double cloth.

\section{b) Center stitched double cloth}

These double cloths are constructed on the principle of center thread stitching. In these cloths, besides a face and back series of threads, there is a third series of threads those are introduced as stitching threads at different intervals. The stitching can be done in warp way, weft way or in both the way. Basically, stitching threads stay in between the face layers and the back layers of the cloth and are visible on the face or back at the stitching points. Figure 3 shows a center stitched double cloth.

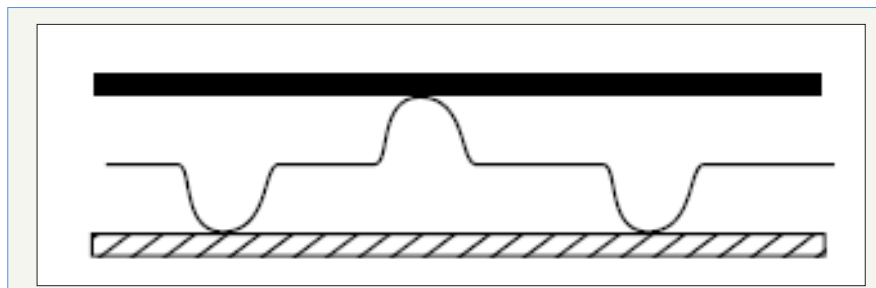

Figure 3: Center stitched double cloth.

\section{c) Double cloth stitched by thread interchange}

It is constructed on the principle of stitching by thread interchange. This fabric is like the self-stitched double cloths as the stitching is done by means of either the face or the back threads themselves. Basically, the dissimilarity is that, a group of face threads interlace or stitch with another group of back threads at regular intervals. An example is given in below Figure 4 . 


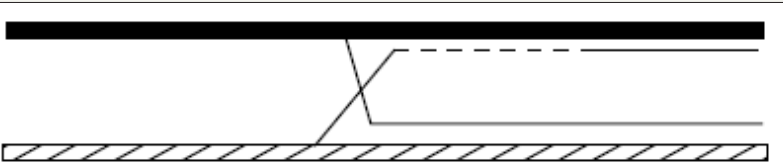

Figure 4: Double cloth stitched by thread interchange.

\section{d) Double cloth stitched by cloth interchange}

This cloth is constructed on the principle of cloth interchange. In these cloths, the cloth layers change places at intervals. The firmness of this type of structural cloth depends on the frequency of the exchange of the face and back layers of the cloth. An example is given in below Figure 5 .

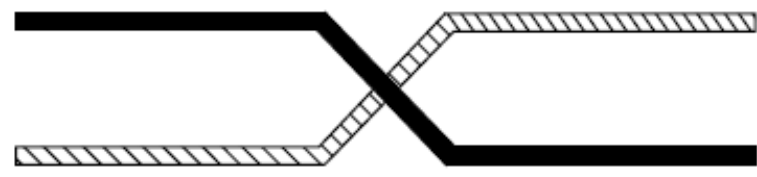

Figure 5: Double cloth stitched by cloth interchange.

\section{e) Alternate single ply and double ply construction}

It is constructed on the principle of alternation as single ply and double ply construction. In these cloths, the group of threads are forming the face are merged together with those of the back to form a single layer at intervals. The face layer is separated from the back, wherever a figure is formed. An example is given in below Figure 6 .

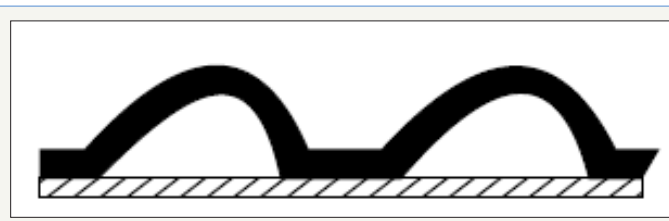

Figure 6: Double cloth based on alternate single ply and double ply construction.

\section{Double cloth without using any stitching thread}

The Figure 7 shows double cloth that is produced without using any stitching threads. These cloths become single cloths after removing it from the loom. In this figure, there is shown a double width cloth and a tubular.

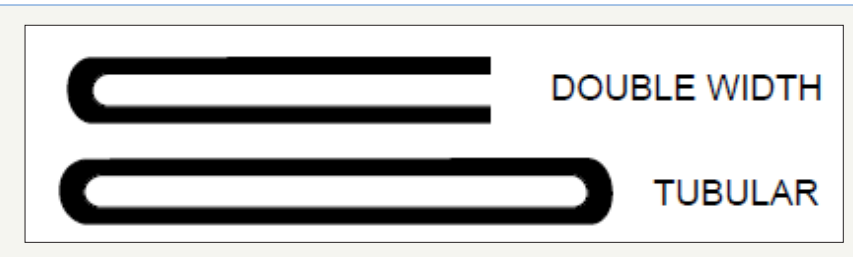

Figure 7: Double cloths without stitching threads.

\section{Construction of self-stitched double cloths}

Methods of self-stitched double cloths are discussed below:

a) Stitching from face to back

b) Stitching from back to face

c) Combination stitching

\section{a) Stitching from face to back}

The face warp stitching with the back weft, so the warp has long overlap on the back side. It is necessary to use weft faced weaves with long warp floats at the back. The back fabric also should have weft faced weave. The long weft floats can be used for stitching with the warp threads of the face fabric. Divided draft is preferable for this system [6].

In the below mentioned Figure 8, face weave is used 4/4 and back weave is used $2 / 2$. Insertion of the face weave on the face ends, face picks only according to the original design and similar that refers to the back weave: insert the back weave on the back ends and picks only, according to the original design. Two separate fabrics are produced one above the other with the final weave.

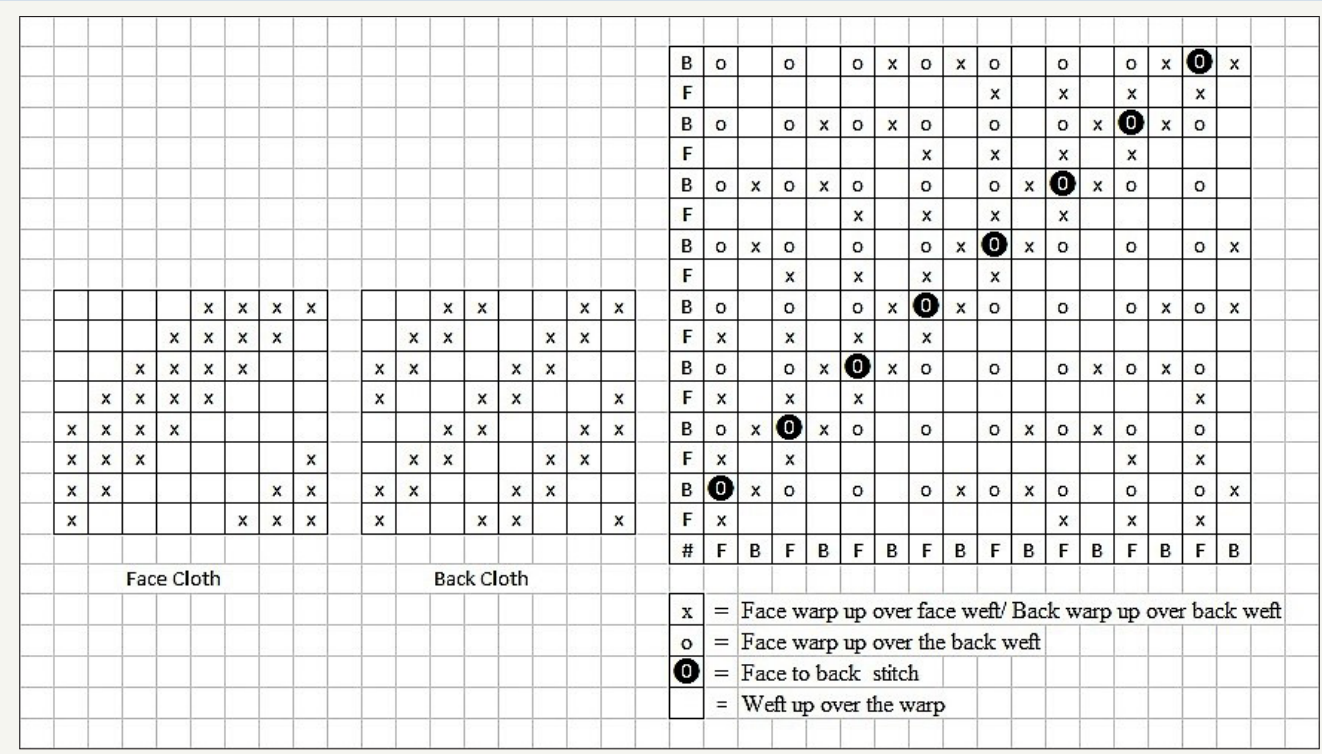

Figure 8: Self-stitched double cloths that Stitches from face to back. 


\section{b) Stitching from back to face}

Here, in this method, the face and back fabrics are stitched together by the back warp to stitch with the face weft. So, the stitching back warp threads are caused to float above the corresponding face weft picks. Various stages of construction of the double cloth constructed by this method. To get this type of stitching, the lower side of the face fabric should have long weft floats and the upper side of the back fabric should have longer warp floats. The stitching point is selected in the middle of the long weft float at the lower side of the face fabric. Divided draft is preferable here, as two series of warp threads are found [7].

In the below mentioned Figure 9, face weave $4 / 4$ and back weave $2 / 2$. Insertion of the face weave on the face ends, face picks only according to the original design and similar that refers to the back weave insertion of the back weave on the back ends and picks only.

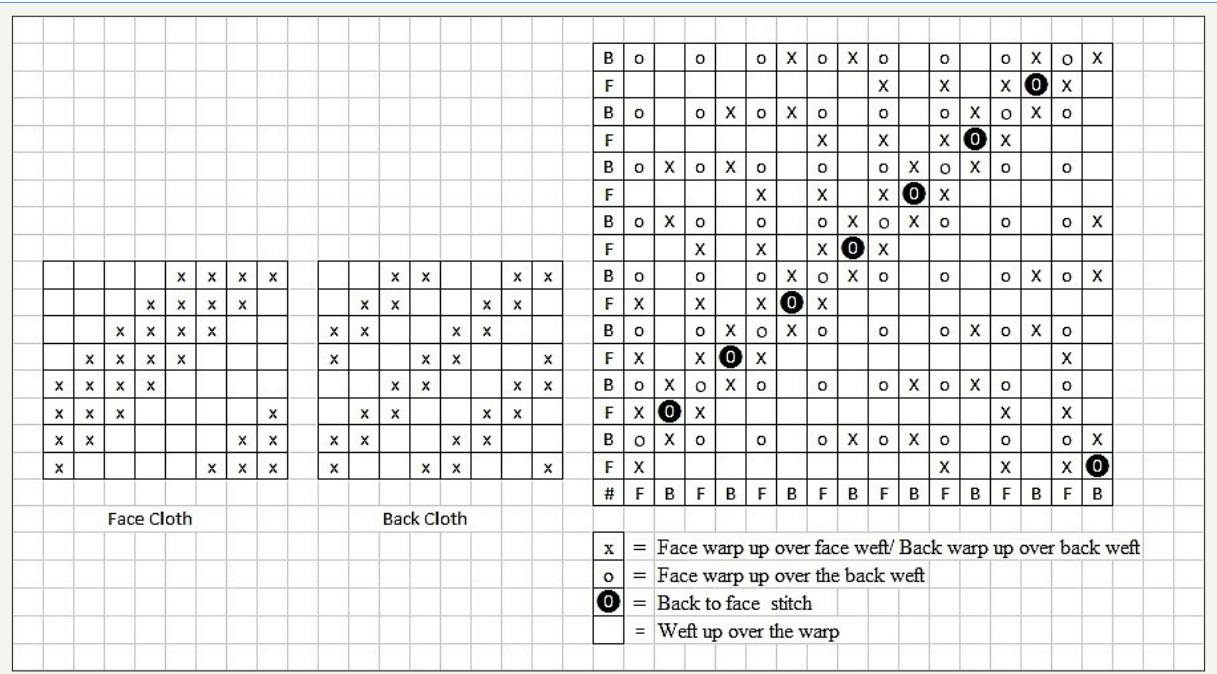

Figure 9: Self-stitched double cloths that Stitches from back to face.

\section{Wadded double cloth}

Wadded yarns are used in double cloth to add extra weight and material in fabric. The threads are basically used in either warp way or weft way. The wadded yarns are coarser than the other threads and are made of cheaper material. The aim of using wadded thread is to increase air permeability or thermal conductivity and comfortability [8].

\section{Double cloth with wadded warp}

In these types of fabrics, the wadding threads are used in warp way. Comparatively, it is a well-situated and inexpensive method. During weaving, greater pressure is introduced on warp threads, which requires the use of a better-quality wadding material. In the warp wadded structures, the wadding ends must be raised on all back picks and left down on all face picks. Use of wadding threads enhance the strength of a double cloth in the direction of the wadding yarn. It is also helpful to increase firmness of the wadding threads those are stitched to the double cloth. These stitches are being placed adjacent to the ordinary stitches to reduce their effect.

In the below mentioned Figure 10, face weave is $4 / 4$ and back weave is $4 / 4$. The complete design shows face and back with wadded yarn in warp direction. In warp wedded structures, the wedding ends are raised on all back picks and left down all face picks.

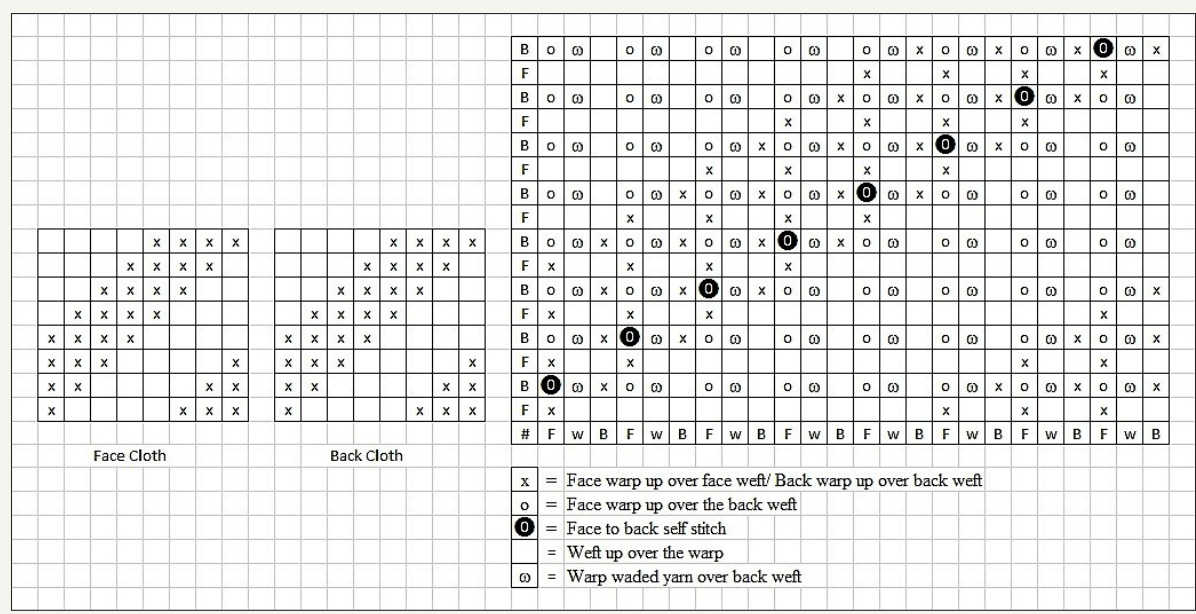

Figure 10: Double cloth with wadded warp. 


\section{Double cloth with wedded weft}

These fabrics are manufactured by inserting wadded threads in weft way. The wadded picks stay in between the face and back picks. Wadding yarn lies in between the two fabrics without interweaving with either, the same conditions are necessary, so far as regards the face weave, the ties and the back weave, as in the construction of double cloths. The wadded design is the same as the ordinary double design except for the inclusion of the wadding threads, and in order that comparisons may be made, the double weave without the wadding. In weft wadded structures all face ends are up, and all back ends are down, on wadding picks.

In the below mentioned Figure 11, face weave is $4 / 4$, back weave is $4 / 4$. The complete weave shows the face and the back weave with wadded threads in weft way. In weft wadded structure, all face ends remain up, all back ends remain down on wedding pick.

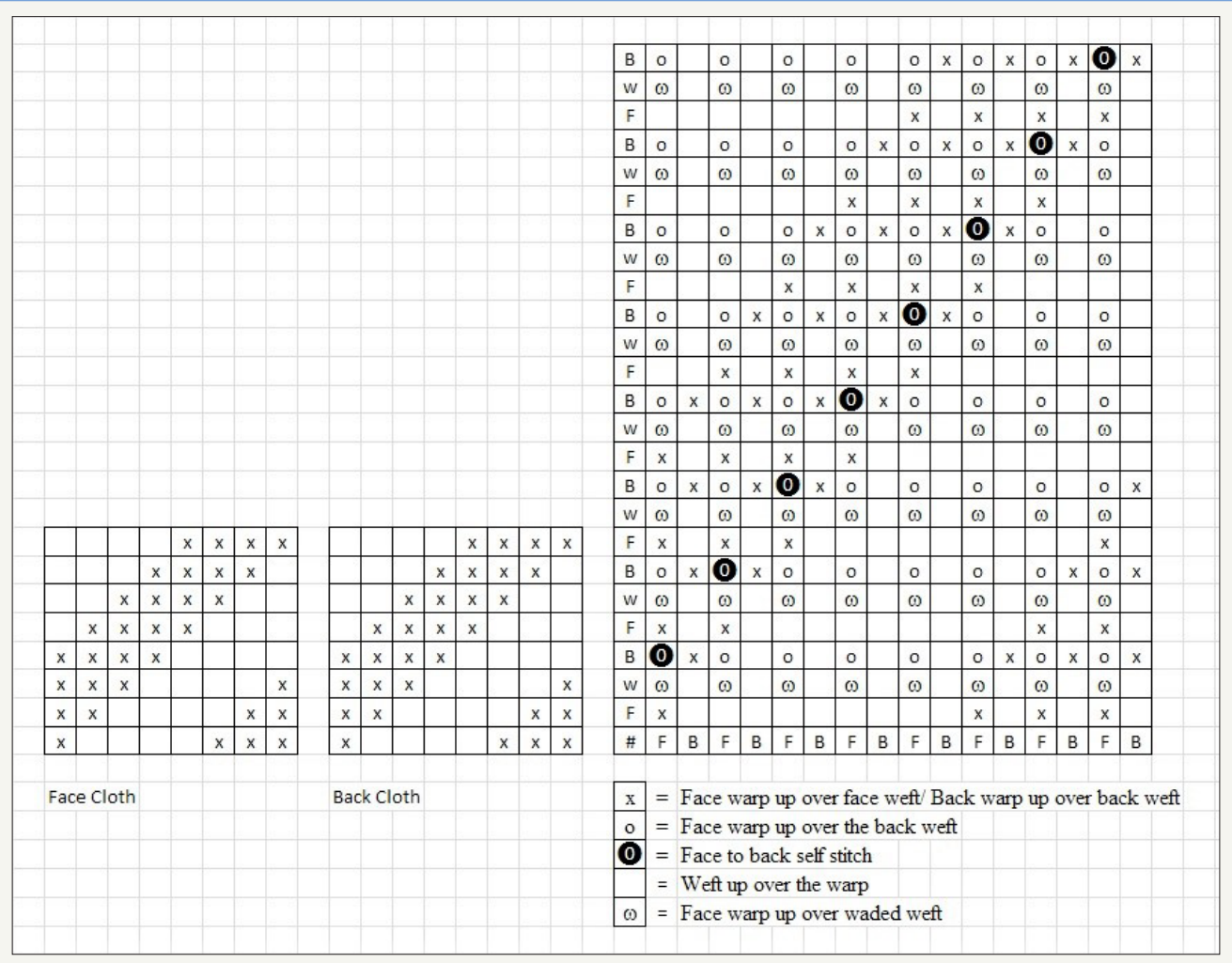

Figure 11: Double cloth with waded weft.

\section{Materials and Method}

\section{Materials}

Six types of double cloths were used in this experiment to do the required tests. Figure 12 is given below that shows a double cloth used in this experiment. In these types of fabrics, 2/2 RHT weave is used as face cloth and $2 / 2$ RHT weave is used as back cloth. The face warp is stitched with the back weft, so the warp should have long overlap on the back side. It is necessary to use weft faced weaves with long warp floats at the back. The back fabric also should have weft faced weave. The long weft floats on the upper side of the back fabric can be used for stitching with the warp threads of the face fabric. Divided draft is used to make this fabric. The construction of this samples are given in the Table 1. Composition, weight and width are also given in Table 1.

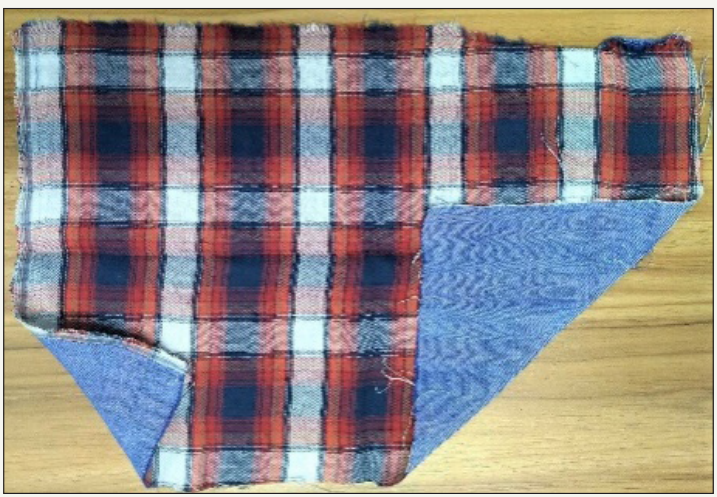

Figure 12: Double cloth fabric, used in this experiment. 
Table 1: Double cloths used in this experiment.

\begin{tabular}{|c|c|c|c|c|}
\hline Sample Type & Composition & Construction & Weight $\left(\mathrm{gm} / \mathrm{m}^{2}\right)$ & Width (") \\
\hline Type 1 & $100 \%$ Cotton & $48 \times 48 / 160 \times 82$ & 144 & 59 \\
\hline Type 2 & $100 \%$ Cotton & $40 \times 40 / 160 \times 82$ & 168 & 59 \\
\hline Type 3 & $100 \%$ Cotton & $36 \times 36 / 160 \times 82$ & 186 & 59 \\
\hline Type 4 & $100 \%$ Cotton & $48 \times 48 / 150 \times 72$ & 133 & 59 \\
\hline Type 5 & $100 \%$ Cotton & $40 \times 40 / 150 \times 72$ & 156 & 59 \\
\hline Type 6 & $100 \%$ Cotton & $36 \times 36 / 150 \times 72$ & 171 & 59 \\
\hline
\end{tabular}

\section{Air permeability test}

An air permeability tester was used to do this experiment. The model of the machine was FX 3300 and manufactured in Switzerland. Test pressure was $125 \mathrm{~Pa}$ and tested area was $38 \mathrm{~cm}^{2}$.
The tests were done using the six types of double cloths mentioned in Table 1. The instrument works in accordance with ASTM D 737 Standard [9]. The unit of measurement is $\mathrm{cm}^{3} / \mathrm{cm}^{2} / \mathrm{s}$. The tests were conducted with above criteria and the results are showed in Figure 13.

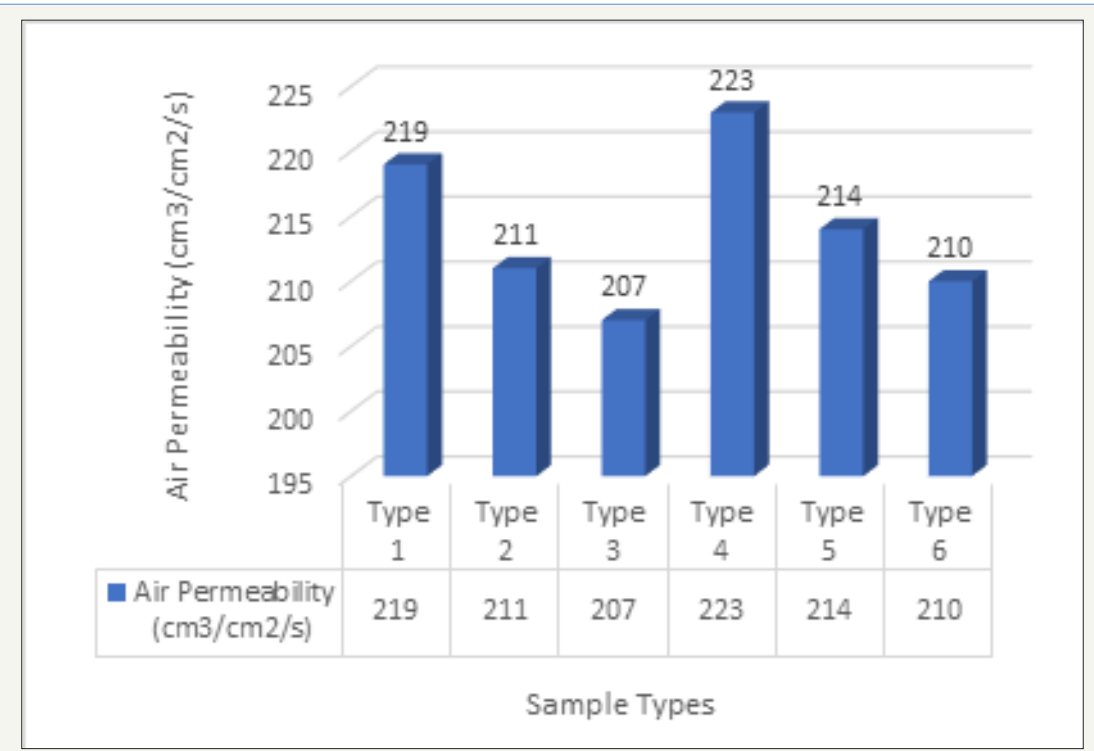

Figure 13: Air permeability test of different types double cloth.

\section{Thermal conductivity test}

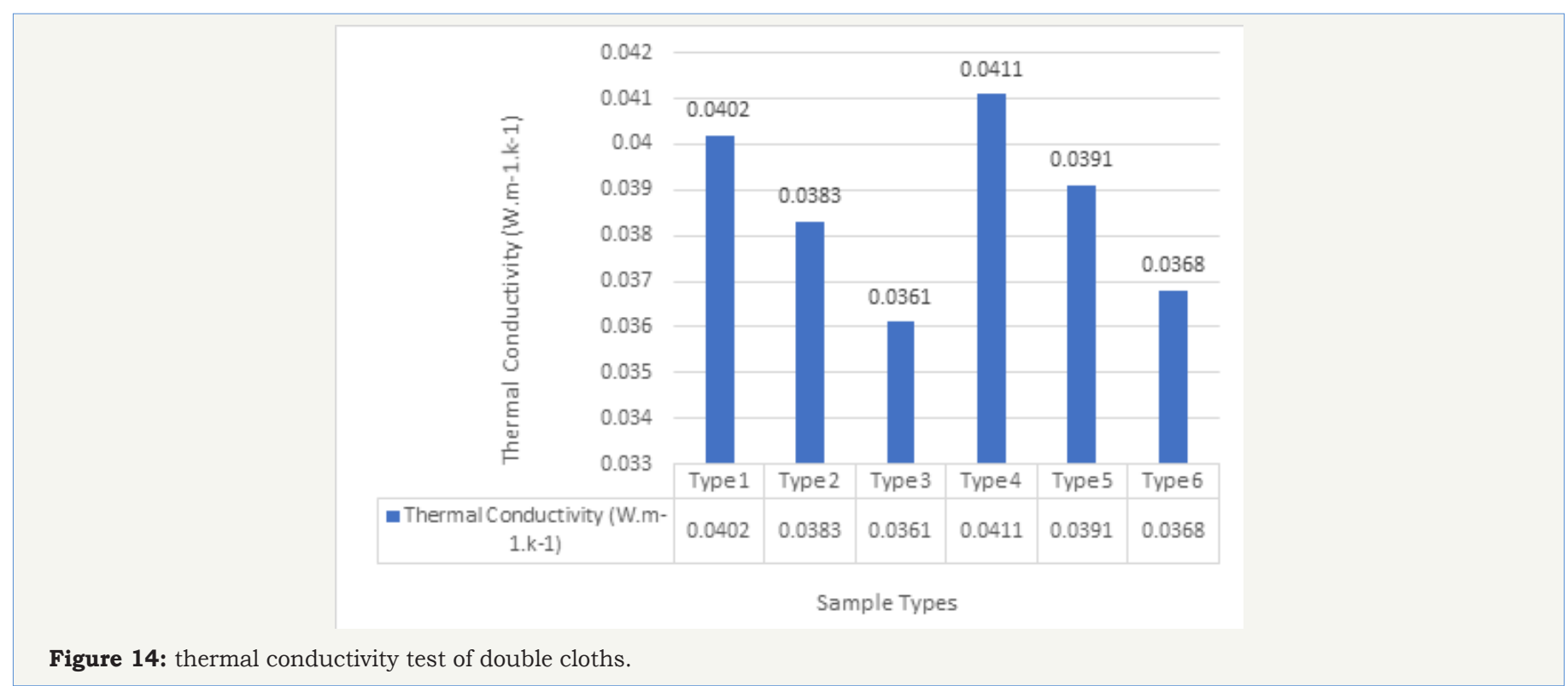


The thermal conductivity is the transfer of heat from one part of a body to another with which it is in contact. Thermal conductivity $\lambda$ is defined as ability of a material to transmit heat and it is measured in watts per square metre of surface area for a temperature gradient of $1 \mathrm{~K}$ per unit thickness of $1 \mathrm{~m}$. Testex TF130 auto thermal conductivity tester [10] was used to measure the thermal conductivity of the fabric at six different cloths in ASTM D1518 standard [11] as mentioned in Table 1. The composition of the fabrics tested were $100 \%$ cotton. Six types of samples were used in this experiment as mentioned in the Table 1 . Six thermal conductivity measurements were made for six fabrics. Ambient temperature for all measurements was $21^{\circ} \mathrm{C}$. Thermal conductivities of fabric is important for designing clothing for different climates. A fabric having a low thermal conductivity is most favorable in colder climates to prevent heat loss from the body; while in warmer climate a fabric with a higher thermal conductivity will allow heat to pass more easily from human body to help cool down. With using above criteria, thermal conductivity was tested in the lab and showed the results in Figure 14.

\section{Shrinkage test}

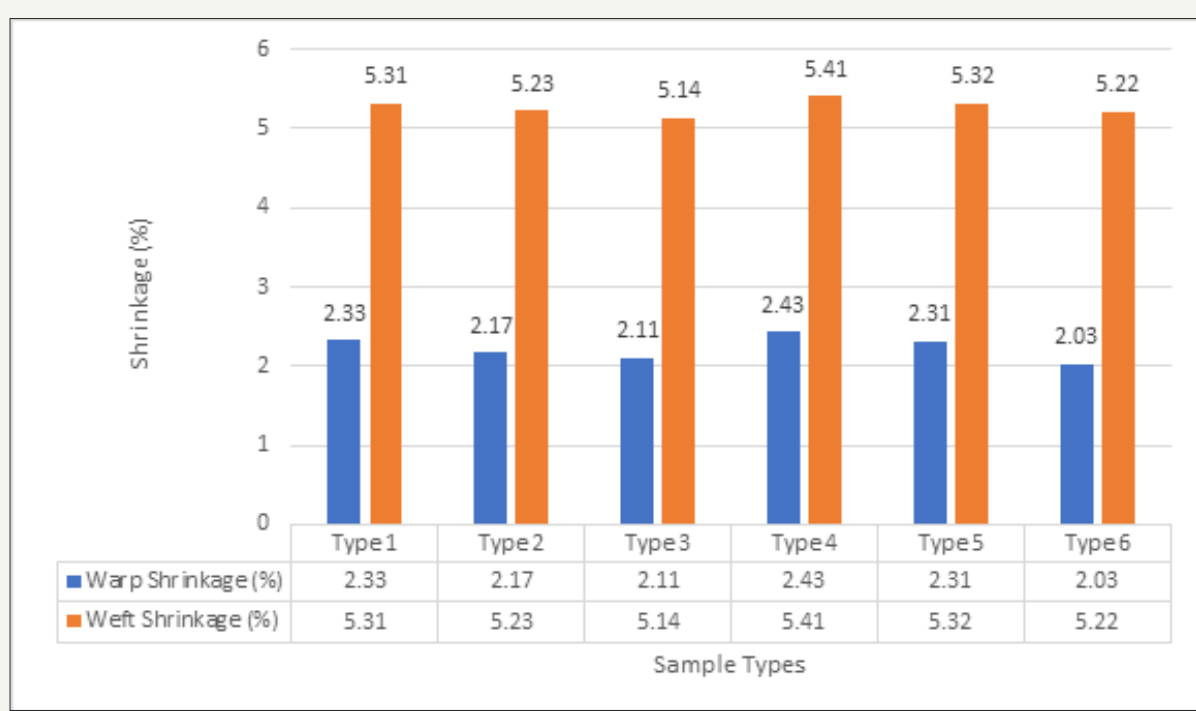

Figure 15: Shrinkage test of double cloth.

The cotton fabric has a tendency to shrink after washing, is called shrinkage. Testex automatic shrinkage tester TF176 was used to test the shrinkage of cloth. Shrinkage rate refers to the percentage that fabrics' size presents shrink when washed. Six different fabrics were used as mentioned in Table 1 , were cut $50 \mathrm{~cm}$ $x 50 \mathrm{~cm}$ area and $48 \mathrm{~cm} \times 48 \mathrm{~cm}$ was marked with permanent marker and interlock stitching was done at the edge of the six cloths for washing and to get shrinkage test reports. ASTM D6207 standard

\section{Tear strength test}

was used to measure the fabric shrinkage [12]. Laundering includes detergent washing, normal rinse wash, extraction and drying. The process steps are- detergent wash for 90 minutes with $60{ }^{\circ} \mathrm{C}$ temperatures, rinse washed with room temperature for 45 minute and then extraction for 15 minute and at last drying for 5 minutes. Finally, shrinkage was measured by getting the length differences due to washing and showed the results in Figure 15.

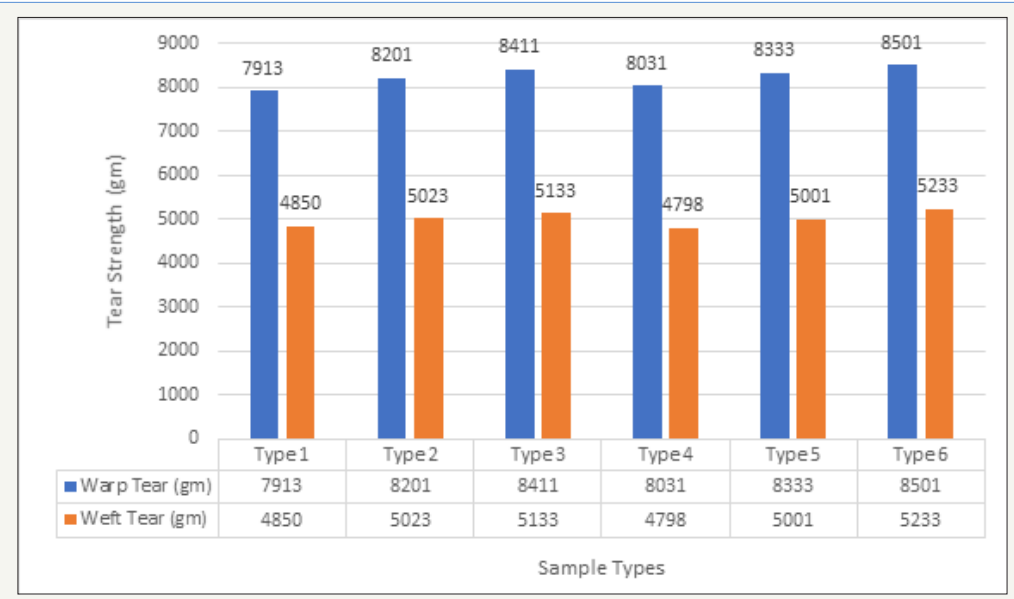

Figure 16: Measurement of tear strength. 
"Instron 5900 tear testing machine" was used to measure the tear strength of this double cloth in ASTM D2261 Standard [13]. "3inch x 8inch" size fabrics were cut from six different types of the cloth as mentioned in Table 1 and put into the instrument for tear testing. The value of this tests were taken in gram. Using above criteria, the results of tear strengths were found and showed the consequences in Figure 16.

\section{Tensile strength test}

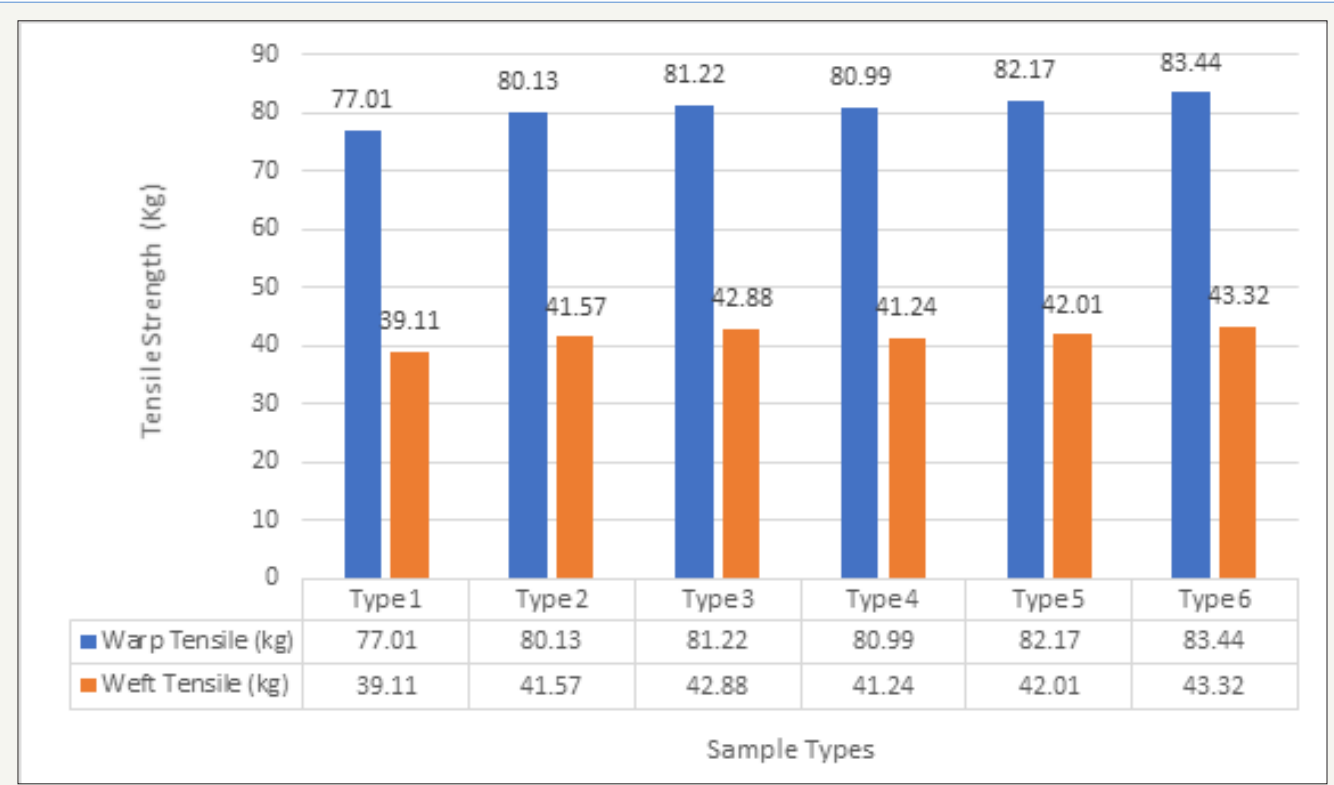

Figure 17: Measurement of tensile strength.

"TESTEX Tensile Testing Machine TF001" was used to measure the tensile strength of the fabric in ASTM D76 Standard [14]. "2.5inch x 8-inch" size fabric was cut from six different types of fabrics as mentioned in the Table 1 and put to the instrument for tensile testing. The value for this test were taken in unit of $\mathrm{kg}$. Using above criteria, the results of tensile strengths were found and showed the consequences in Figure 17.

\section{Result and Discussion}

\section{Results of the air permeability test}

For doing air permeability testing of the different types of samples showed different results. For the sample type 1, which was cut from the double cloth of a particular portion exposed the value of $219 \mathrm{~cm}^{3} / \mathrm{cm}^{2} / \mathrm{s}$ and showed the results in the Figure 13. For sample type 2 , the value was $211 \mathrm{~cm}^{3} / \mathrm{cm}^{2} / \mathrm{s}$, for sample type 3 , the value was $207 \mathrm{~cm}^{3} / \mathrm{cm}^{2} / \mathrm{s}$, for sample type 4 , the value was $223 \mathrm{~cm}^{3} /$ $\mathrm{cm}^{2} / \mathrm{s}$, for sample type 5 , the value was $214 \mathrm{~cm}^{3} / \mathrm{cm}^{2} / \mathrm{s}$. for sample type 6 , the value was $210 \mathrm{~cm}^{3} / \mathrm{cm}^{2} / \mathrm{s}$. It was seen that; values were different for different types of samples. It is seen for the Figure 13 that; higher air permeability was obtained for fabric of looser construction as type 1 from the Table 1 . The loose the construction is, the more the value for air permeability is. The least value of air permeability was obtained for the fabric of most compact structure among the six, because compact construction disallows more air to pass therefore air permeability is less for denser qualities.

\section{Results of thermal conductivity}

For doing thermal conductivity testing of the different types of samples showed different results. For the sample type 1, which was cut for the double cloth of a particular portion exposed the value of thermal conductivity 0.0402 W.m-1.k-1 and showed the results in the Figure 14. For sample type 2, the value was found 0.0383 W.m$1 . \mathrm{k}-1$, for sample type 3 , the value was found $0.0361 \mathrm{~W} . \mathrm{m}-1 . \mathrm{k}-1$, for sample type 4 , the value was found 0.0411 W.m-1.k-1, for sample type 5 , the value was found 0.0391 W.m-1.k-1.For sample type 6, the value was found 0.0368 W.m-1.k-1. It is seen from the Figure 14 that, lower thermal conductivity was obtained for the fabric of denser construction, and higher thermal conductivity value was obtained for the fabric of looser construction. Higher thermal conductivity value will allow heat to pass more easily from our body, so that faster cool down is possible. For the more compact fabrics, the thermal conductivity value is less meaning, heat will not pass so fast from fabrics.

\section{Results of shrinkage}

For doing shrinkage testing of the different samples showed different results. For sample type 1, which was cut for the double cloth of a portion exposed the value of warp shrinkage $-2.33 \%$ and weft shrinkage $-5.31 \%$ and showed the results in the Figure 15 . For sample type 2 , the value was found warp shrinkage $-2.17 \%$ and weft shrinkage $-5.23 \%$. For sample type 3 , the value was found warp shrinkage $-2.11 \%$ and weft shrinkage $-5.14 \%$. For sample type 4 , the value was found warp shrinkage $-2.43 \%$ and weft shrinkage $-5.41 \%$. For sample type 5 , the value was found warp shrinkage $-2.31 \%$ and weft shrinkage $-5.32 \%$. For sample type 6 , the value was found warp shrinkage $-2.03 \%$ and weft shrinkage $-5.22 \%$. It is seen from the Figure 15 that; more shrinkage values were obtained for the fabrics of looser constructions and less shrinkage values were obtained for the fabrics of compact constructions. Compact constructions shrink less when it come in contact of water, on 
the other hand, loose constructions shrink more when it come in contact of water.

\section{Results of tear strength}

For doing tearing testing of the different types of samples showed different results. For the sample type 1, which was cut for the double cloth of a particular portion exposed the value of warp tear 7913gram and weft tear 4850 gram and showed the results in the Figure 16. For sample type 2, the value was found warp tear 8201gram and weft tear 5023gram. For sample type 3, the value was found warp tear 8411gram and weft tear 5133gram. For sample type 4 , the value was found warp tear $8031 \mathrm{gram}$ and weft tear 4798gram. For sample type 5, the value was found warp tear 8333gram and weft tear 5001gram.For sample type 6, the value was found warp tear 8501 gram and weft tear 5233. It is seen from the Figure 16 that, higher tear strengths are obtained for the fabrics of heavier weight and lower tear strengths are obtained for the fabrics of lesser weights. It is clear that, heavier fabrics have more tear strength.

\section{Results of tensile strength}

For doing tensile testing of the different types of samples obtained different results. For sample type 1, which was cut for the double cloth of a portion exposed the value of warp tensile $77.01 \mathrm{~kg}$ and weft tensile $39.11 \mathrm{~kg}$ and showed the results in the Figure 17. For sample type 2 , the value was found warp tensile $80.13 \mathrm{~kg}$ and weft tensile $41.57 \mathrm{~kg}$. For sample type 3 , the value was found warp tensile $81.22 \mathrm{~kg}$ and weft tensile $42.88 \mathrm{~kg}$. For sample type 4 , the value was found warp tensile $80.99 \mathrm{~kg}$ and weft tensile $41.24 \mathrm{~kg}$. For sample type 5, the value was found warp tensile $82.17 \mathrm{~kg}$ and weft tensile $42.01 \mathrm{~kg}$. For sample type 6 , the value was found warp tensile $83.44 \mathrm{~kg}$ and weft tensile $43.32 \mathrm{~kg}$. It is seen from the Figure 17 that; higher tensile strengths are obtained for the fabrics of heavier weight and lower tensile strengths are obtained for the fabrics of lesser weights. It is clear that, heavier fabrics have more tensile strength.

\section{Conclusion}

At last it can be concluded that, double cloth is a type of advanced weave with an advanced construction that creates two textiles, one above the other by means of at least two sets of warps and two sets of weft. A woven fabric construction made by interlacing two or more sets of warp yarns with two or more sets of filling yarns. Double weave is a family of weave styles in which the face of the fabric is effectively detached from the back except at specific connecting interlacing, yet with each side maintaining sufficient individual structural integrity to be identified as distinct fabrics in itself. These fabrics are met in both decorative and utilitarian roles and inhabit interesting excesses at either end of those spectra. Double cloth fabrics were produced by self-stitching, center stitching and by thread interchange which all were discussed in this project. Construction of Self-stitched double cloths can be produced by stitching from face to back, stitching from back to face and by combination stitching all were discussed briefly in this project. Double cloth fabric can also be produced by waded yarns in both warp and weft way. Higher air permeability obtained for fabric of looser construction useful for the body. In case of more compact fabrics, thermal conductivity value was less so that heat will pass slowly for this fabric types. Shrinkage values are less for the fabrics of denser constructions. Higher strengths obtained for the fabrics of heavier weight. Specially, air permeability and thermal conductivity can be improved significantly through the production of double cloths.

\section{References}

1. Barber EJW (1991) Prehistoric textiles: The development of cloth in the neolithic and bronze ages with special reference to the aegean. Princeton University Press, Princeton NJ, USA

2. American Fabrics and Fashion Editors (1980) Encyclopedia of textiles. ( $3^{\text {rd }}$ edn), Englewood Cliffs, Prentice-Hall, New Jersey, USA.

3. Oelsner GH, Samuel SD (1875) A handbook of weaves. Dover Publications, Mineola, New Jersey, USA.

4. Collier B, Tortora P (2001) Understanding textiles. Prentice-Hall Inc., Upper Saddle River, New Jersey, USA.

5. Gioello DA (1982) Understanding fabrics: from fiber to finished cloth. Fairchild Publishers, New York, USA, p. 101.

6. Shah Alimuzzaman Belal (2009) Understanding textiles for a merchandiser. BMN3 Foundation, Dhaka, Bangladesh, pp. 244-253.

7. Grosicki ZJ (1975) Watson's textile design and colour, (7 ${ }^{\text {th }}$ edn) Woodhead Publishing, UK, pp. 301-380.

8. Gokerneshan N (2004) Fabric structure and design. New age international publishing limited. Daryaganj, New Delhi, India, pp. 110152.

9. ASTM D 737 Standard (2011) Air permeability test method of fabrics, ASTM International, West Conshohocken, 1: 19-25.

10. Testex TF130 auto thermal conductivity tester, NO. 3, Daliantang Industrial Dt, China.

11. ASTM D1518 standard (2010) Thermal conductivity of fabrics. ASTM International, West Conshohocken 1: 41-43.

12. ASTM D6207 standard (2009) Shrinkage testing of fabrics. ASTM International, West Conshohocken 1: 63-66.

13. ASTM D2261 Standard (2013) Standard test methods for tear strength properties of fabrics. ASTM International, West Conshohocken 1: 34-37.

14. ASTM D76 standard (2014) Standard test methods for tensile strength properties of fabrics. ASTM International, West Conshohocken 5: 11-13. 
Creative Commons Attribution 4.0 International License

For possible submissions Click Here

Submit Article

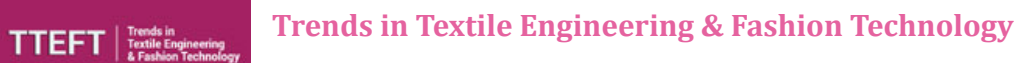 Benefits of Publishing with us}

- High-level peer review and editorial services

- Freely accessible online immediately upon publication

- Authors retain the copyright to their work

- Licensing it under a Creative Commons license

- Visibility through different online platforms 